\title{
Structural and Surface Morphology Analysis of Nickel Phthalocyanine Thin Films
}

\author{
Mohammed T. Hussein, Eman M. Nasir, Addnan H. Al-Aarajiy \\ Department of Physics, University of Baghdad, Baghdad, Iraq \\ Email: eman.itabi@gmail.com
}

Received January 23, 2013; revised March 13, 2013; accepted April 15, 2013

Copyright (C) 2013 Mohammed T. Hussein et al. This is an open access article distributed under the Creative Commons Attribution License, which permits unrestricted use, distribution, and reproduction in any medium, provided the original work is properly cited.

\begin{abstract}
The thin films of Nickel Phthalocyanine (NiPc) on glass substrates were prepared by vacuum evaporation at different substrates temperatures $(300,325,350,400,450) \mathrm{K}$. The structure and surface morphology of NiPc in powder and thin film forms $(265 \mathrm{~nm})$ were studied using X-ray diffraction and atomic force microscope (AFM), and showed that there was a change and enhance in the crystallinity and surface morphology due to change in the substrates temperatures. Analysis of X-rays diffraction patterns of NiPc in powder form showed that it had an $\alpha$-polycrystalline phase with monoclinic system with lattice constants $\mathrm{a}=1.513 \mathrm{~nm}, \mathrm{~b}=0.462 \mathrm{~nm}, \mathrm{c}=2.03 \mathrm{~nm}$ and $\beta=123.46^{\circ}$. Thermal evaporation of NiPc at different substrates temperatures led to $\beta$-crystalline films oriented preferentially to the (100) plane with different substrate temperatures. The mean crystallite size increased with substrates temperatures from $300 \mathrm{~K}$ to $450 \mathrm{~K}$. This result was supported by AFM measurements, which exhibited a relatively larger grain size.
\end{abstract}

Keywords: Nickel Phthalocyanine; Organic; XRD; AFM; Morphology; Thin Film; Structure

\section{Introduction}

The materials considered of most importance are the phthalocyanines in the organic dyes and pigments. Phthalocyanines have found applications as semiconducting devices, photovoltoic cells, rectifying devices, liquid crystal displays and gas sensors. The electrical, optical and structural properties of phthalocyanine thin films are dependent on various parameters such as evaporation rate, substrate temperature and post-deposition annealing [1]. Phthalocyanines are found in different phases i.e. $\alpha-, \beta$ and $\gamma$-phases. Metal-substituted and metal free phthalocyanines are readily used as active layer in several applications such as gas sensors, photocapacitive and photoresistive detectors, Organic Thin Film Transistors (OTFTs), colour filters and organic laser materials. Among the various metal-substituted phthalocyanines, nickel phthalocyanine has received considerably less attention. In the present study, we report the structural properties of nickel phthalocyanine thin films prepared at different substrates temperatures. To explore the potentially interesting properties of the material, structural and morphological characterization is thus considered as a prerequisite for deep insight knowledge of the material. These structural properties mostly depend on the deposition technique, the heat-treatment temperature and the condi- tions during film deposition. Annealing process is a broadly employed method to enhance the quality of the crystal and exploit structural defects in the material. During thermal annealing, the morphology and structural properties of the material change [2-4]. For semiconductor devices like light emitting diodes the structural properties are very important, therefore, it is essential to investigate how thermal annealing and substrate temperatures process affects these properties $[5,6]$. In the present work, thin films of NiPc have been fabricated on glass substrates by vacuum evaporation techniques. A detailed study on the effects of heating substrate temperatures on the structural properties and surface morphology has been performed by X-ray diffraction and atomic force microscopy (AFM). Hence, the purpose of our work is to find the optimal temperature for which the film produces best structural properties.

\section{Experimental}

The NiPc powder used in this study was obtained from Aldrich chemical company and was used as the source material for thermal evaporation. Its molecular structure is shown in Figure 1. Before starting the deposition, the glass substrate was cleaned in an ultrasonic bath for 10 min using acetone, followed by rinsing in distilled water. 


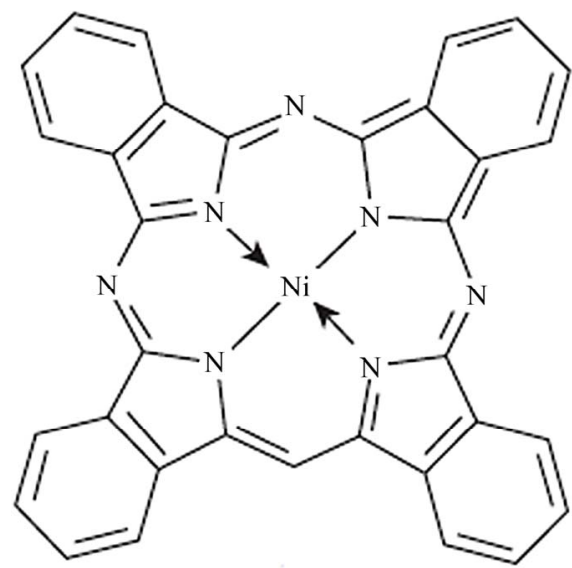

Figure 1. Molecular structure of Ni-phthalocyanine (NiPc).

The substrate was dried in open air in a cleaned room. A molybdenum boat was used as a heating source. Thin films of NiPc, were deposited by vacuum evaporation technique on thoroughly cleaned glass substrate at different substrate temperatures $(300,325,350,400,450) \mathrm{K}$ using Edwards 306 system. During deposition, the pressure in the vacuum chamber was kept constant at about $10^{-5}$ mbar and the deposition rates of all the films were kept at about $0.2 \mathrm{~nm} / \mathrm{s}$, Also the substrate are placed at a distance of $15 \mathrm{~cm}$ from the source. The thickness of the films was measured using Michelson interferometer technique. The thickness of the films was $265 \mathrm{~nm}$. The adhesion of the films to the substrate seems to be extremely good. The structural analysis of films is performed by $\mathrm{X}$-ray diffractometer with $\mathrm{Cu} \mathrm{K} \alpha$ radiation $(\lambda=1.54 \AA)$. The surface morphology of the NiPc films at different substrate temperatures $\left(T_{s}\right)$ is investigated by means of atomic force microscopy (AFM).

The lattice parameters of the films were calculated using the Bragg's formula:

$$
2 d \sin \theta=n \lambda
$$

The grain size of the crystallites were calculated from the XRD using Scherer's relation,

$$
D=K \lambda / \beta \cos \theta
$$

Where $K=0.94$ is a constant, $\lambda$ the wavelength of $\mathrm{X}$-ray, $\beta$ the full width half maximum and $\theta$ the diffraction angle. The micro strain $(\varepsilon)$ and the dislocation density $(\delta)$ of films were estimated using the equations

$$
\begin{gathered}
\mathcal{E}=\beta \cos \theta / 4 \\
\delta=1 / D^{2}
\end{gathered}
$$

\section{Results and Discussions}

The most common polymorphic crystal phases occur in Ni-phthalocyanines are $\alpha$, and $\beta$, that have two crystallite structures. The $\alpha$ phase is metastable and obtained either as a polycrystalline powder or as a thin film deposited on a cold substrate in vacuum [7]. The phase $\beta$ can be obtained in single crystal form or as a thin film formed by deposition of phthalocyanine on heated substrates, which are thermodynamically stable. Phase separation could be responsible for the de gradation of the optical properties. The $\alpha$ form is metastable and can be converted to $\beta$ form by temperature treatments with structures tetragonal and monoclinic respectively. The differences in the $\mathrm{d}$ values are attributed to higher X-ray absorption, sample purity, particle size, preferred orientation and crystal texture $[4$, 7].

The X-ray diffraction of the NiPc powder used as the source material for sublimation is given in Figure 2. The peaks are identified using the standard JCPDS File No. 11-0744 data. The preferential orientation is found to be in the (100) direction at $2 \theta=6.938^{\circ}$ and that it has an $\alpha$ crystalline phase with monoclinic system with lattice constants $\mathrm{a}=1.513 \mathrm{~nm}, \mathrm{~b}=0.462 \mathrm{~nm}, \mathrm{c}=2.03 \mathrm{~nm}$ and $\beta$ $=123.46^{\circ}$. The close agreement showed that the powder is in the monoclinic structure as reported for other phthalocyanines [1-7]. Table 1 gives the lattice constant of present observed and the standard data of NiPc.

X-ray diffraction pattern of NiPc thin film of thickness $(265 \mathrm{~nm})$ prepared at different substrate temperatures $(300,325,350,400,450) \mathrm{K}$ are shown in the Figure 3 respectively. XRD pattern indicates that all the samples are single phase and that it has a $\beta$-crystalline phase [4-8]. The difference between $\beta$ - and $\alpha$-phases is attributed to the tilt angle of b-axis of the unit cell. The structure of the NiPc thin film is determined as tetragonal with preferential orientation along the (100) direction. Well-defined diffraction peaks by (100) gives the direction of the preferential orientation as deposited film (JCPDS, File No. 11-0744). The preferential orientation is at $2 \theta=6.859^{\circ}$. The spacing between the planes, $\mathrm{d}=12.876 \AA$. The $\mathrm{d}$ values obtained from diffractogram are in perfect match with JCPDS record. The diffraction peaks are well matched with the previous observations [1-8]. The increased crystallinity is attributed to the destruction of pseudomorphic layers present in the film upon high substrate temperature. Table 2 shows the structure parameters of NiPc films, The full width at half maximum (FWHM) intensity of preferential orientation, shows that the FWHM are reduced with increasing substrate temperatures, which represents better lattice quality [1,8-10].

From the Table 2 it is observed that the grain size increases which intern decreases strain and dislocation density. The grain size $D$ of the film is calculated using the Scherrer relation, the grain size increases from (22.2 $71.8 \mathrm{~nm})$ for substrate temperatures $(300-450 \mathrm{~K})$. This is because the pseudomorphic layers formed at room temperature, which are in the metastable state, are destroyed by increasing the substrate temperatures and lar- 


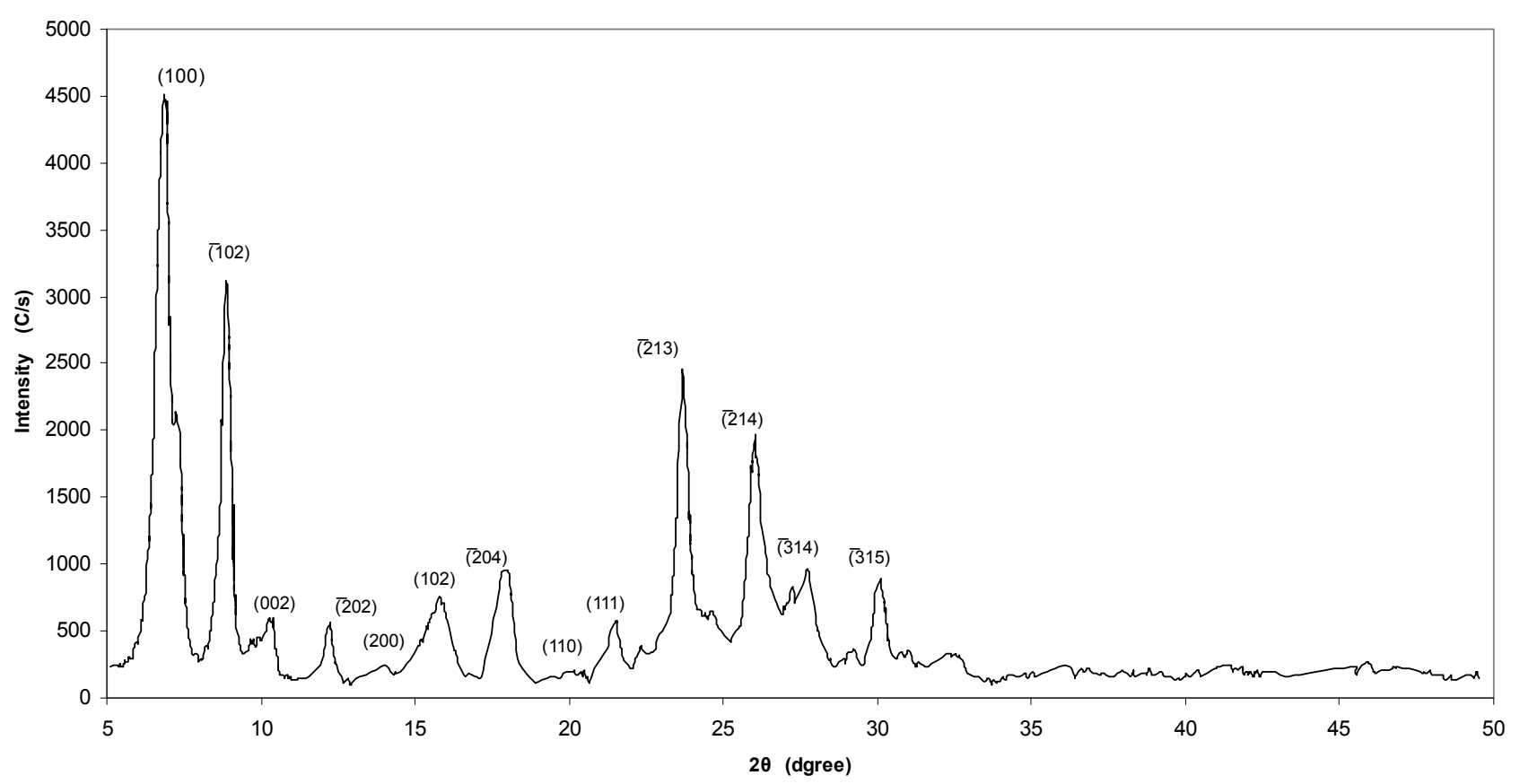

Figure 2. X-ray diffraction of the NiPc powder.

Table 1. The present observed and the standard data NiPc.

\begin{tabular}{cccc}
\hline Lattice constant & Present work & Pcpdf & Error ratio \% \\
\hline $\mathrm{a}(\AA)$ & 15.128 & 14.9 & 1.53 \\
$\mathrm{~b}(\AA)$ & 4.621 & 4.7 & 1.6 \\
$\mathrm{c}(\AA)$ & 20.355 & 19.9 & 2.28 \\
$\beta(\mathrm{deg})$ & 123.46 & $121.15(\mathrm{deg})$ & -1.9 \\
$\mathrm{~d}(\AA)$ & 7.370124 & 7.266181 & -1.43 \\
\hline
\end{tabular}

ger crystallites are formed or this can be attributed to the onset of phase transition occurring in NiPc. Also this may be attributed to a phase transition or to the regrowth of phthalocyanine. This mean that the crystallization of the films is a function of substrate temperatures and the optimal temperature for which the film produces best structural properties is at $400-450 \mathrm{~K}$. Also from Figure 3 two distinct peaks (200) and (314) are visible at high diffraction angles for heating substrate 400 and $450 \mathrm{~K}$ respectively, and this is agreement with other literatures [6-9], and this is may be attributed to enhancement and recrystallization these films by increasing the substrate temperatures.

Surface morphology of NiPc thin film is shown in Figures 4 and 5 which prepared at different substrate temperatures $(300,325,350,400,450) \mathrm{K}$. It shows that the morphology of the NiPc thin film has larger number of grain size and are homogeneously distributed, which indicates the crystalline nature of the film. It is obvious because the films were prepared at different substrate temperatures, due to the heating grain growth will takes place which gives the crystallinity and good surface morphology [2]. These results are in good agreement with XRD characteristics. The crystal morphology and molecular orientation change with substrate temperature. The average grain size increases as temperature increases. Gas sensitivity depends both up on material parameters, such as the crystal phase of the material and film parameters, such as the surface topography of the film. These structural characterizations confirm that the substrate temperature allows us to control the structural organization of nickel phthalocyanine molecules in their solid state, from which a large improvement of the properties can be expected. Table 3 shows that the grain size increased by increasing the substrate temperature.

The morphology of these films, has also confirmed the appearance of polycrystallites and increasing grain size when substrate temperature is raised. This is caused due to the nucleating behavior of the substance [7]. The root mean square (rms) roughness of the film at different substrate temperatures are shown in Table $\mathbf{3}$ which increases with increasing $\mathrm{T}_{\mathrm{s}}$. The best results can be seen for the film prepared at heating substrate of $400-450 \mathrm{~K}$. The $\mathrm{NiPc}$ thin films, after increasing substrate temperatures to $400-450$, show relatively rough and non-uniform surface, suggesting the formation of interpenetrating molecular network of NiPc. $400-450 \mathrm{~K}$ and above, deterioration feature is observed due to degradation of the film. From the application point of view, the higher efficiency devices are obtained from the film of greater roughness.

\section{Conclusions}

Thin films of NiPc were fabricated by the thermal 

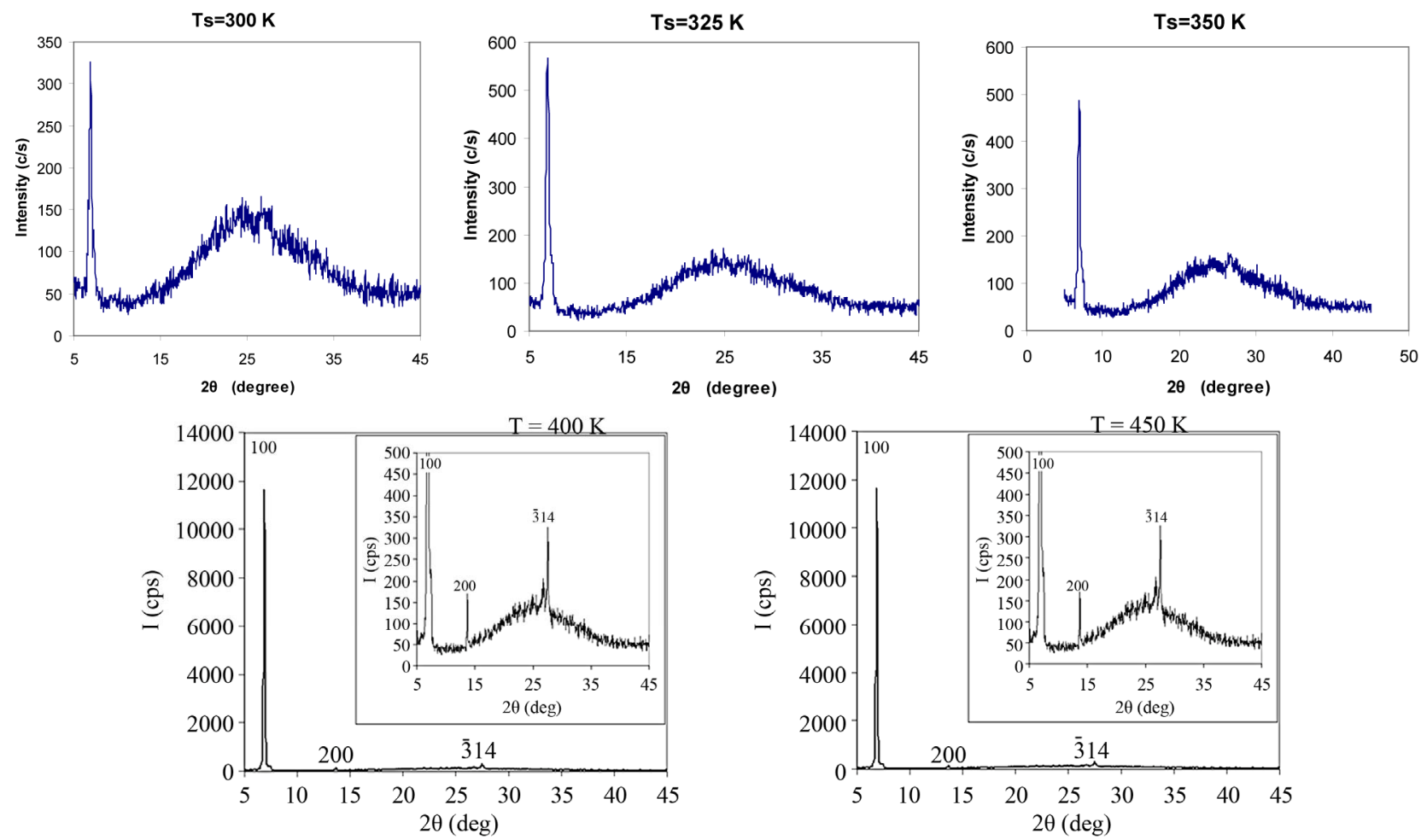

Figure 3. X-ray diffraction pattern of NiPc thin film at different substrate temperatures $(300,325,350,400,450) \mathrm{K}$.

Table 2. Shows the structure parameters of NiPc films.

\begin{tabular}{cccccccc}
\hline $\mathbf{T}_{\mathbf{s}}(\mathbf{k})$ & $\mathbf{( h k l )}$ & $\mathbf{2 \theta}(\mathbf{d e g r e e})$ & $\mathbf{d}(\mathbf{\AA})$ & $\mathbf{F W H M}(\mathbf{d e g r e e})$ & $\mathbf{D}(\mathbf{n m})$ & $\boldsymbol{\varepsilon} \times \mathbf{1 0}^{-\mathbf{3}}$ & $\boldsymbol{\delta} \times \mathbf{1 0}^{-\mathbf{1 4}} \mathbf{l i n e s} / \mathbf{m}^{\mathbf{2}}$ \\
\hline 300 & 100 & 7.060 & 12.51 & 0.3741 & 22.2 & 1.62 & 20.2 \\
325 & 100 & 7.028 & 12.56 & 0.3746 & 22.19 & 1.63 & 20.3 \\
350 & 100 & 7.030 & 12.56 & 0.3583 & 23.2 & 1.55 & 18.5 \\
400 & 100 & 6.920 & 12.76 & 0.1115 & 45.0 & 0.485 & 1.79 \\
$\mathbf{4 5 0}$ & 100 & 6.990 & 12.63 & 0.1157 & 71.8 & 0.503 & 1.93 \\
\hline
\end{tabular}

evaporation technique on glass substrates with thickness $(265 \mathrm{~nm})$ prepared at different substrate temperatures successfully. The outcome of this investigation can be summarized as follows:

- X-ray diffraction patterns obtained for NiPc powder showed $\alpha$-polycrystalline phase with monoclinic system, while the thin film of thermally evaporated NiPc showed it has a single crystalline $\beta$-phase film oriented preferentially (100) plane as tetragonal. It can be seen that the intensity, $d$ value and sharpening of the main peak increase with substrate temperature which shows crystallization of the films when substrate temperature increases.

- Diffraction peaks obtained for the films prepared at higher substrate temperatures are sharp and that is due to the increased crystallinity.

- The full width at half maximum (FWHM) intensity of preferential orientation shows that the FWHM are reduced with increasing substrate temperatures and the grain size increases which intern decreases strain and dislocation density, which represents better lattice quality.

- The grain size increases from $(22.2-71.8 \mathrm{~nm})$ for substrate temperatures $(300-450 \mathrm{~K})$, this can be attributed to the onset of phase transition occurring in NiPc. Also this may be attributed to a phase transition or to the regrowth of phthalocyanine. This mean that the crystallization of the films is a function of substrate temperatures and the optimal temperature for which the film produces best structural properties is at $400-450 \mathrm{~K}$.

- Rearrangements of molecules are taking place with different substrate temperatures. The change in grain size shows that the properties of NiPc are controlled by its structure and morphology depending on increases the substrate temperatures.

- By analyzing the AFM topographic images, we found that there is a significant influence of substrate temperatures on the surface morphology of NiPc films and the crystal morphology and molecular orientation 

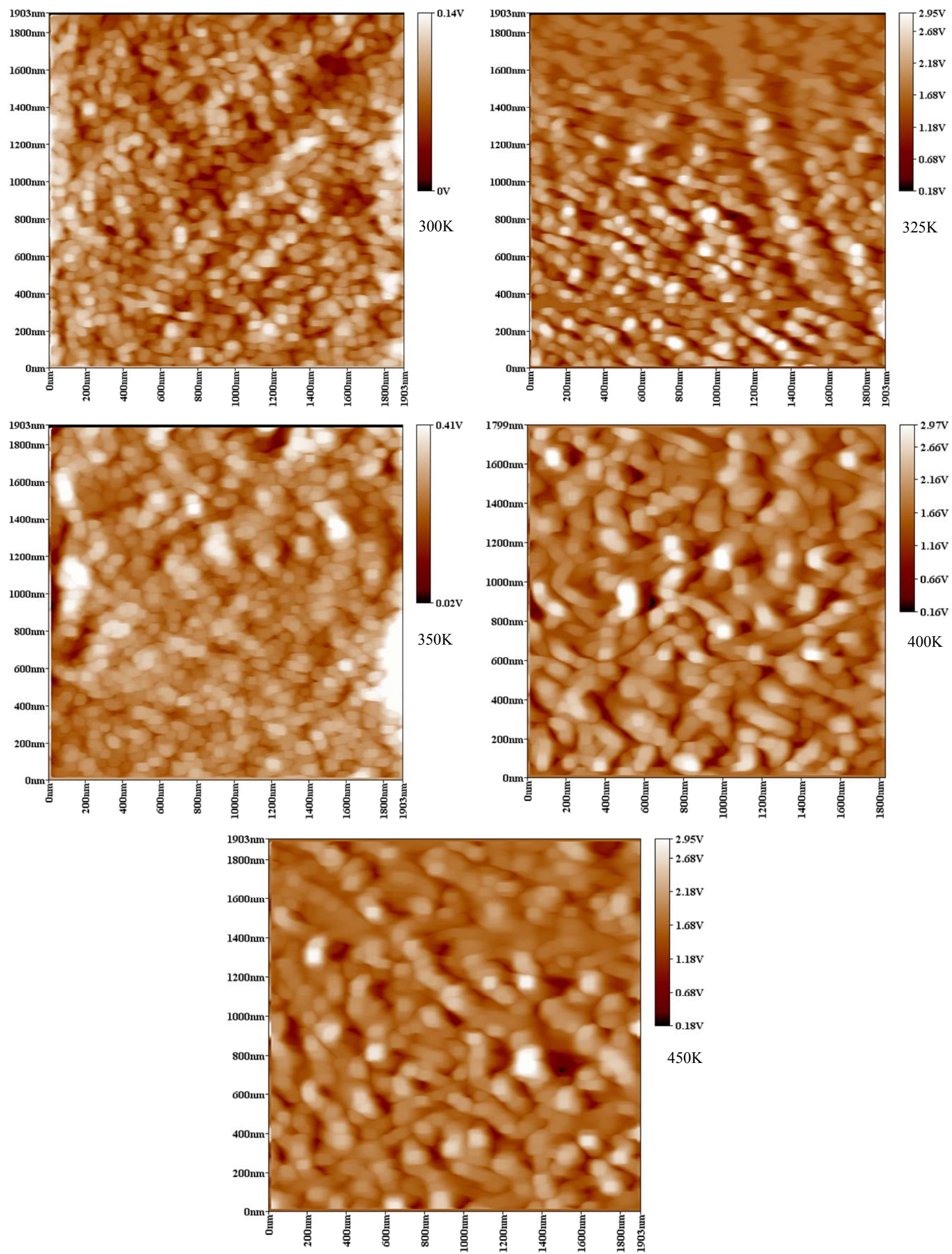

Figure 4. 2-D AFM images of NiPc thin films at different substrate temperatures $(300,325,350,400,450) \mathrm{K}$. 

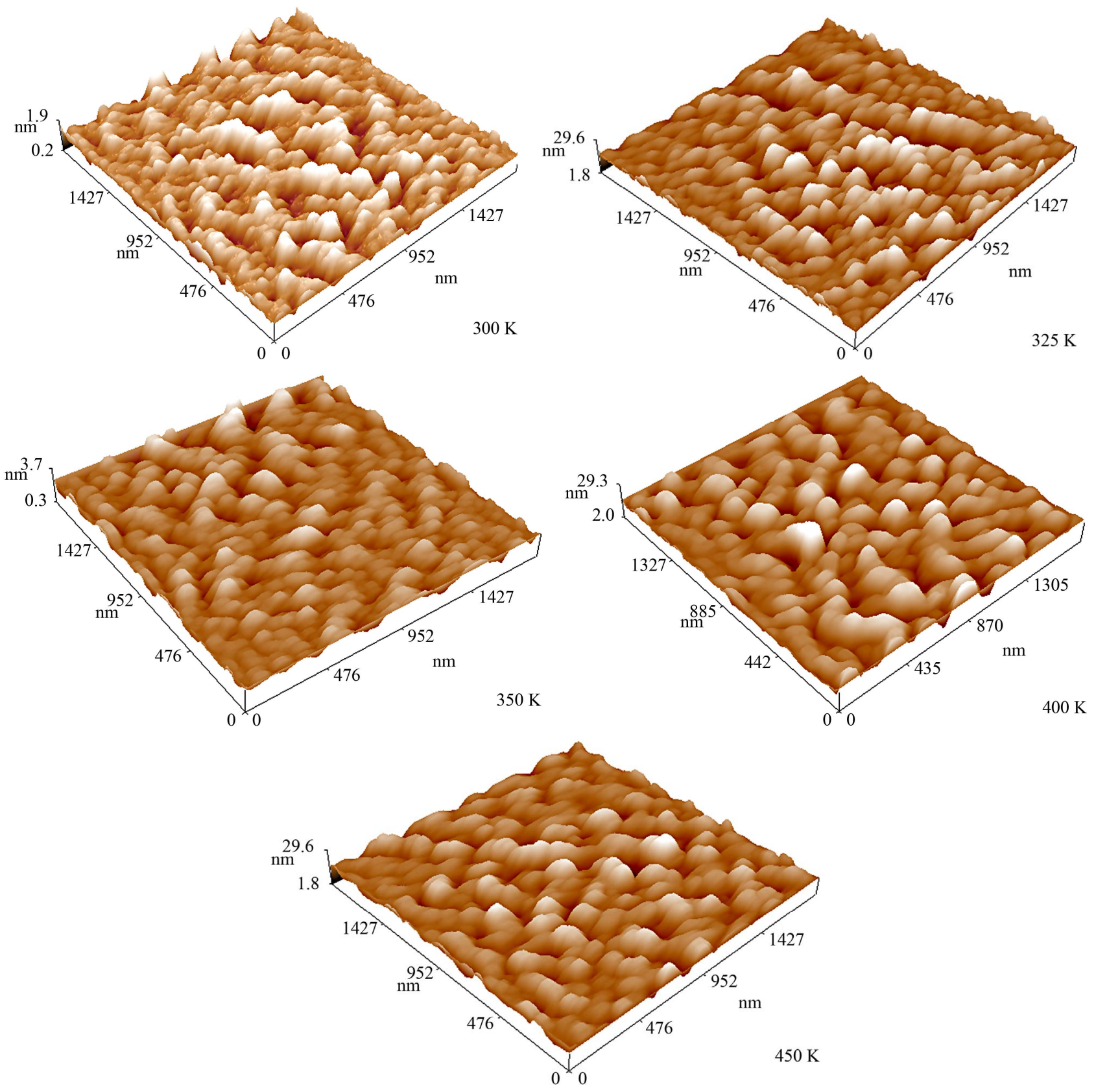

Figure 5. 3-D AFM images of NiPc thin films at different substrate temperatures $(300,325,350,400,450) \mathrm{K}$.

Table 3. Shows the grain size and roughness of NiPc.

\begin{tabular}{ccc}
\hline Ts $(\mathbf{k})$ & Roughness Average $(\mathbf{n m})$ & $\mathbf{D}(\mathbf{n m})$ \\
\hline 300 & 0.216 & 92.42 \\
325 & 0.275 & 108.18 \\
350 & 2.9 & 94.04 \\
400 & 2.86 & 116.26 \\
450 & 2.54 & 116.59 \\
\hline
\end{tabular}

change with substrate temperature. These films showed a larger number of grain size, homogeneously distributed, rough which indicates the crystalline nature of the film. The surface roughness of Ni-Pc thin films is increased by increasing substrate temperatures, which makes it useful for higher efficiency devices. The increase in the temperature beyond $400 \mathrm{~K}$ has higher roughness and grain size.

- These structural characterizations confirm that the substrate temperature allows us to control the structural organization of nickel phthalocyanine molecules in their solid state, from which a large improvement of the properties by increasing the substrate temperatures, therefore, the best results can be seen for the film prepared at heating substrate of $400-450 \mathrm{~K}$. 
These results are in good agreement with XRD characteristics.

\section{Acknowledgements}

The work was supported by Department of Physics college of Science, thin films and opto-electronic laboratories, Baghdad University.

\section{REFERENCES}

[1] K. N. N. Unni and C. S. Menon, "Electrical, Optical and Structural Studies on Nickel Phthalocyanine Thin Films," Materials Letters, Vol. 45, No. 6, 2000, pp. 326-330. doi:10.1016/S0167-577X(00)00127-0

[2] F. Aziza, K. Sulaimana, M. R. Muhammad, M. H. Sayyad and Kh. Karimov, "Influence of Thermal Annealing on the Structural Properties of Vanadyl Phthalocyanine Thin Films: A Comparative Study," World Academy of Science, Engineering and Technology, Vol. 56, 2011, pp. 852-854.

[3] D. Gu, Q. Y. Chen, J. P. Shu, X. D. Tang, F. X. Gan, S. Y. Shenb, K. Liu and H. J. Xu, "Optical Recording Performance of Thin Films of Phthalocyanine Compounds," Thin Solid Films, Vol. 257, No. 1, 1995, pp. 88-93. doi:10.1016/0040-6090(94)06327-3

[4] S. Shihub and R. Gould, "Structure and Phase-Change Phenomena in Evaporated Thin Films of Cobalt Phthalo- cyanine," Physica Status Solidi A, Vol. 139, No. 1, 1993, pp. 129-138. doi:10.1002/pssa.2211390110

[5] A. El-Bosaty, et al., "Surface Plasmon-Cobalt Phthalocyanine Sensor for $\mathrm{NO}_{2}$ Gas," Egypt. J. Solids, Vol. 29, No. 1, 2006, pp. 121-129.

[6] M. Shah, Kh. S. Karimov and M. H. Sayyad, "Organic Semiconductor Nickel Phthalocyanine-Based Photocapacitive and Photoresistive Detector," Semiconductor Science and Technology, Vol. 25, No. 7, 2010, pp. 7501475018. doi:10.1088/0268-1242/25/7/075014

[7] B. Joseph and C. S. Menon, "Studies on the Optical Properties and Surface Morphology of Nickel Phthalocyanine Thin Films," E-Journal of Chemistry, Vol. 4, No. 2, 2007, pp. 255-264. doi:10.1155/2007/643834

[8] P. Kalugasalama and S. Ganesanb, "Surface Morphology of Annealed Lead Phthalocyanine Thin Films," International Journal of Engineering Science and Technology, Vol. 2, No. 6, 2010, pp. 1773-1779.

[9] Z. G. Ji, K. W. Wongb, P. K. Tseb, R. W. M. Kwoka and W. M. Laub, "Copper Phthalocyanine Film Grown by Vacuum Deposition under Magnetic Field," Thin Solid Films, Vol. 402, No. 1-2, 2002, pp. 79-82. doi:10.1016/S0040-6090(01)01702-3

[10] P. Kalugasalam and S. Ganesanb, "Structural Analysis of Annealed Lead Phthalocyanine Thin Films," International Journal of Engineering Science and Technology, Vol. 2, No. 7, 2010, pp. 2567-2573. 